\title{
Topological and Metagenomic Evidence For Intensified Bacterial-Fungal Interactions in Mangrove Root Interior
}

\section{Zhengyuan Zhou}

Sun Yat-Sen University https://orcid.org/0000-0002-2653-775X

\section{Ruiwen Hu}

Sun Yat-Sen University

\section{Yanmei Ni}

Guangdong Agribusiness Agriculture Institute

Wei Zhuang

Sun Yat-Sen University

\section{Zhiwen Luo}

Sun Yat-Sen University

\section{Weiming Huang}

Sun Yat-Sen University

\section{Qiuping Zhong}

Sun Yat-Sen University

\section{Qingyun Yan}

Sun Yat-Sen University

Zhili He

Sun Yat-Sen University

Cheng Wang ( $\square$ wangcheng5@mail.sysu.edu.cn)

Sun Yat-Sen University https://orcid.org/0000-0002-3712-9890

\section{Research}

Keywords: Network, Bacterial-fungal interaction, Endosphere, Quorum sensing, Cobamide

Posted Date: February 26th, 2021

DOI: https://doi.org/10.21203/rs.3.rs-237640/v1

License: (c) (1) This work is licensed under a Creative Commons Attribution 4.0 International License.

Read Full License 


\section{Abstract}

Background: Plant roots host a repertoire of bacteria and fungi, whose ecological interactions could improve their functions and plant performance. However, potential interactions and underlying mechanisms remain largely unknown in root-associated microbial communities at a continuous finescale.

Results: We analyzed microbial intra- and inter-domain network topologies, keystone taxa, and interaction-related genes across four compartments (non-rhizosphere, rhizosphere, episphere and endosphere) from a soil-mangrove root continuum, using amplicon and metagenome sequencing technologies. We found that both intra- and inter-domain networks displayed notable differences in the structure and topology across four compartments. Compared to three peripheral compartments, the endosphere was a distinctive compartment with more intensive interactions in bacterial-fungal network than in bacterial or fungal network, which could be related to three bacterial keystone taxa (Vibrio, Anaerolineae and Desulfarculaceae) detected in the endosphere as they are known to intensify interdomain interactions with fungi and stimulate biofilm formation. Also, high abundances of genes involved in cell-cell communications by quorum sensing ( $r h l l$, lasl, pqsH and lasR) and aerobic cobamide biosynthesis ( $c o b G, c o b F$ and $c o b A$ ) were detected in the endosphere.

Conclusions: Our results reveal intensified inter-domain interactions of endophytes in the mangrove roots, creating a distinct micro-environment to promote a biofilm life-style.

\section{Background}

The roots of soil-grown plants host diverse microbial communities, and microbe-microbe interactions (e.g. inhibition, facilitation and competition) are highly prevalent and have emerged as an important feature of plant root ecosystems [1, 2]. Recent studies have shown that intra- or inter-domain microbial interactions could sustain the harmony of biogeochemical processes and keep the nutritional status and ecological balance for plant health [3, 4]. One typical example is the association between arbuscular mycorrhizal fungi and nitrogen-fixing bacteria, and their synergistical interactions can result in better nutrient uptake and higher plant productivity compared to plants symbiotic with either of them alone [5]. At the community level, inter-domain microbial interactions were specifically enriched with negative correlations between bacteria and filamentous eukaryotes in Arabidopsis thaliana roots, where bacterial communities were essential for plant survival and protection against filamentous eukaryotes. These studies contribute to the growing body of evidence that the plant growth and health are closely connected to root-associated microbial interactions [2], which carry out key functions like synergistic effects on plant growth, maintenance of host-microbiota balance, and protection against environmental pathogens [2].

Current studies suggested that the root microhabitat could be divided into four continuous compartments: non-rhizosphere (buck soil), rhizosphere [6], episphere and endosphere [2]. Microbial communities in each compartment were shown to exhibit unique characteristics $[2,7]$. The rhizosphere 
could selectively gather specific bacterial and fungal populations via root exudates, which conversely exerted a marginal influence on microbes in the non-rhizosphere soil $[8,9]$. Compared to the rhizosphere, the episphere played a more critical role for the controlled entry of specific microbes into the plant root, leading to the selective enrichment of Proteobacteria and the absence of soil-derived fungi in the endosphere $[2,10]$. In fact, the significant variations that root-associated bacterial and fungal communities underwent along the soil-root continuum were irrespective of plant hosts, and microbial diversity inside plant roots was much lower than that in other three exterior root compartments [7]. These findings have greatly advanced our understanding of root-associated bacterial and fungal community structure and assembly. However, the intra- and inter-domain interactions of root-associated microbial communities and mechanisms along the soil-root continuum are poorly understood.

In order to unravel the organization and strength of microbial interactions, network analysis has been widely used as a promising tool to disentangle microbial co-abundance in various environments, such as groundwater, oceans and soil environments [11]. Recently, it has been applied to evaluate the microbemicrobe associations in plant roots $[12,13]$ and their responses to environmental parameters $[14,15]$. More importantly, the topology-based network analysis provides an opportunity to identify the keystone taxa, which are strongly interconnected and have an important effect on communities $[3,16]$. In despite of numerically inconspicuous, keystone taxa confer higher biotic connectivity to the microbial community and therefore can be predictors of community shifts and compositional turnover [17]. However, it has not been well studied whether and how microbe-microbe interaction dynamics act on root-associated microbial communities at a continuous fine scale, arising a pertinent question whether soil-root interface would display a selective role in regulating intra- and inter-domain microbial interactions and determining the profile of keystone taxa.

Many secondary metabolites are usually bioactive and can perform key functions in microbial interactions. A well-established mechanism of cell-to-cell communications is quorum sensing, which is defined to be a stimuli-response system related to cellular density $[18,19]$. Early studies have shown that quorum sensing can regulate bioluminescence and biofilm formation by releasing a specific acylated homoserine lactone (HSL) signaling molecule, which is known as an autoinducer produced by LuxHike proteins [18], and distinct microbes can produce the same type of signaling molecules, which have a role in both inter- and intra-domain interactions [20]. Similarly, cobamides are recognized as mediators of microbial interactions [21,22], making contributions to the nucleotide biosynthesis and catabolism of carbon sources [22]. It is important to note that, microbes of all domains need cobamides, but many of them rely on the surrounding species to complement. This facilitates the ubiquitous establishment of a network of cobamide-dependent interactions in natural ecosystems [22]. Many previous studies have investigated the profile of genes associated with quorum sensing and cobamide biosynthesis in the complex microbial communities; however, little is known about whether and how these genes are connected to the microbe-microbe interaction dynamics at a community level.

As an ecologically important ecosystem, mangrove plays a critical role in carbon storage, climate mitigation, shoreline protection and nutrient filtering [23]. Increasing evidences have revealed a high 
diversity of bacterial and fungal communities inhabiting in the mangrove root [24], and determined their importance for mangrove growth and function $[4,25,26]$. For instance, biological nitrogen fixation performed by diazotrophic bacteria in the vicinity of mangrove roots accounted for $40-60 \%$ of the total nitrogen required by mangroves $[25,27]$. Rhizosphere fungi not only displayed ligninolytic, cellulolytic, and amylolytic activity [4], but also could help mangroves adapt to the waterlogged and nutrient-restricted environments [26]. Despite the improved understanding of functional capabilities of root-associated bacterial and fungal communities, our knowledge of potential interactions and underlying mechanisms within mangrove root-associated microbial communities remains largely unknown.

In this study, we profiled bacterial and fungal communities across four compartments (non-rhizosphere, rhizosphere, episphere, and endosphere) of mangrove roots with the aim of inferring the structure of intraand inter-domain microbial networks and exploring putative keystone taxa along the soil-root continuum. Amplicon and metagenome sequencing were performed to examine network topology and profiles of quorum sensing genes, cobamide biosynthesis genes and microbes in mangrove root-associated microbial communities. This study uncovers how diverse taxonomic groups of bacteria and fungi can form metacommunity-scale networks of intra- and inter-domain microbial interactions, providing a basis for understanding complex spatial processes of plant-microbe systems and engineering complex microbial consortia with predictable behaviors and robust outcomes.

\section{Methods}

\section{Site selection, sampling and environmental properties}

The location of the sampling site is at the Shuidong Bay of Maoming City $\left(21^{\circ} 30^{\prime} 38.82^{\prime \prime} \mathrm{N}\right.$, $\left.111^{\circ} 0^{\prime} 37.27^{\prime \prime} \mathrm{E}\right)$, Guangdong, China. In April 2019, we collected 12 individual mangrove saplings, and divided their root-associated samples into four compartments: non-rhizosphere soil (Non), rhizosphere soil (Rhi), root episphere (Epi) and root endosphere (Endo). These compartments were prepared as described by Duran et al [2] and Edwards et al [7]. The non-rhizosphere soil was shaken off the root, whereas the rhizosphere soil was the part of the soil at $\sim 1 \mathrm{~mm}$ thickness around the root and was washed with sterile water. The clean roots were subsequently washed three times to remove the remaining soil and placed into $1 \times$ TE buffer supplemented with $0.1 \%$ Triton X-100 in a $50 \mathrm{~mL}$ Falcon tube. Next, we collected the episphere samples by washing and extensive shaking in $1 \times$ TE buffer supplemented with $0.1 \%$ Triton X-100. The microbial biomass of episphere was obtained via filtering the resulting suspension through $0.22 \mu \mathrm{M}$ pore size membranes (Nuclepore, Whatman, Meterstone, UK). To capture the endosphere microbial biomass, the roots were surface-sterilized for $1 \mathrm{~min}$ in $80 \%$ ethanol and then sterilized again for $1 \mathrm{~min}$ in $0.25 \% \mathrm{NaClO}$. Samples from all four root-associated compartments were stored at $-80^{\circ} \mathrm{C}$ until DNA extraction.

\section{DNA extraction, PCR amplification and sequencing}

For each of 12 mangrove saplings, approximately $0.5 \mathrm{~g}$ of non-rhizosphere and rhizosphere soil was used for DNA extraction using a Power Soil DNA Isolation Kit following the protocol provided by the 
manufacturer (MoBio, Carlsbad, CA, USA) with the modified sodium dodecyl sulfate extraction method [28]. The episphere compartment DNA was extracted using a Power Water DNA Isolation Kit following the protocol provided by the manufacturer (MoBio, Carlsbad, CA, USA). For the endosphere samples, DNA was extracted from plant samples with the Power Plant DNA Isolation Kit, according to the manufacturer's protocol (Mo Bio Laboratories, Inc., Carlsbad, CA, USA) after thorough grinding with liquid nitrogen. The DNA quality of four root-associated compartments was assessed by Nano Drop ND-2000 Spectrophotometer (Thermo Fisher Scientific, MA, USA) based on 260/280 and 260/230 nm ratios [29]. Qualified DNA samples were diluted to $2 \mathrm{ng} / \mu \mathrm{L}$ for subsequent PCR amplification.

The V3-V4 region of 16S rRNA genes was amplified with the primer pair (forward primer, 5'ACTCCTACGGGAGGCAGCA-3'; reverse primer, 5'- GGACTACHVGGG TWTCTAAT-3') [30] and the ITS1 region of fungal ITS genes were amplified with the primer pair (forward primer, 5 'CTTGGTCATTTAGAGGAAGTAA-3'; reverse primer, 5'-GCTGCGTTCTTCATCGATGC-3') [31]. PCR amplification was conducted in a total volume of $50 \mu \mathrm{L}$ containing $10 \mu \mathrm{L}$ buffer, $0.2 \mu \mathrm{L}$ Q 5 high-fidelity DNA polymerase, $10 \mu \mathrm{L}$ high GC enhancer, $1 \mu \mathrm{L}$ dNTP, $10 \mu \mathrm{M}$ of each primer and $60 \mathrm{ng}$ microbial community DNA. Thermal cycling conditions were as follows: an initial denaturation at $95^{\circ} \mathrm{C}$ for $5 \mathrm{~min}$, followed by 15 cycles at $95^{\circ} \mathrm{C}$ for $1 \mathrm{~min}, 50^{\circ} \mathrm{C}$ for $1 \mathrm{~min}$ and $72{ }^{\circ} \mathrm{C}$ for $1 \mathrm{~min}$, with a final extension at 72 ${ }^{\circ} \mathrm{C}$ for $7 \mathrm{~min}$. The PCR products from the first step PCR were purified with VAHTSTM DNA Clean Beads, and the second round PCR was performed in a $40 \mu \mathrm{L}$ reaction containing $20 \mu \mathrm{L} 2 \times$ Phusion HF MM, $8 \mu \mathrm{L}$ $\mathrm{ddH}_{2} \mathrm{O}, 10 \mu \mathrm{M}$ of each primer and $10 \mu \mathrm{L}$ PCR products from the first step. Thermal cycling conditions were as follows: an initial denaturation at $98^{\circ} \mathrm{C}$ for $30 \mathrm{~s}$, followed by 10 cycles at $98^{\circ} \mathrm{C}$ for $10 \mathrm{~s}, 65^{\circ} \mathrm{C}$ for $30 \mathrm{~s}$ min and $72{ }^{\circ} \mathrm{C}$ for $30 \mathrm{~s}$, with a final extension at $72^{\circ} \mathrm{C}$ for $5 \mathrm{~min}$. All PCR products were quantified by Quant-iT $T^{T M}$ dsDNA HS Reagent and pooled together. High-throughput sequencing analysis of bacterial rRNA genes and fungal ITS genes was performed on the purified, pooled samples using the Illumina Hiseq 2500 platform ( $2 \times 250$ paired ends) at Biomarker Technologies Corporation, Beijing, China.

\section{Sequence analysis of 16S rRNA and ITS gene amplicons}

Raw sequences were trimmed using Trimmomatic [32] and FLASH [33], with a moving window of 50-bp and the quality threshold score of 30. After the singleton elimination, paired 16S rRNA amplicon sequences were then clustered into operational taxonomic units (OTUs) by UPARSE [34] based on a $97 \%$ sequence identity using QIIME's (QIIME: Quantitative Insights into Microbial Ecology) [35] open reference OTU picking strategy with the Greengenes 16S rRNA database (v.13.5) as a reference [36]. Bacterial OTU was represented as OTUB, and fungal OTU was represented as OTUF. We eliminated the sequences matching "Chloroplast" and "Mitochondria" from the datasets. ITS sequences were processed by ITSx [37] and clustered at a $97 \%$ sequence identity by UPARSE [34]. Fungal OTUs were checked for chimeric sequences using the Uchime reference against a dedicated chimera detection database [38], which was based on the UNITE database for fungi identification.

\section{Network construction and analysis}


Microbial intra- and inter-domain networks were constructed based on bacterial and fungal OTU relative abundances across each root compartment. Covariations were measured across 12 biological replicates to create each network. Only OTUs detected in 8 out of 12 replicate samples were used for network construction. Random matrix theory (RMT) was used to automatically identify the appropriate similarity threshold (St) before network construction. St defines the minimal strength of the connections between each pair of nodes [39]. Global network properties were characterized as described in Deng et al. [40]. All analyses were performed with the Molecular Ecological Network Analyses (MENA) Pipeline (http://ieg2.ou.edu/MENA/) [40] and the network structures were visualized using Cytoscape 3.6.1 [41] and Gephi 0.9.2-beta [42].

\section{Module detection and node role identification}

We examined network modularity for each network constructed in this study. A module is a set of nodes (OTUs) that are highly connected inside the group but few connected outside the group [43]. Modules were identified using the greedy modularity optimization method in this study. Modularity $(M)$ is an indicator that measures the extent of network module division, and $M>0.4$ is used as the threshold to define module structure [43]. Additionally, the connectivity of each node was determined according to its within-module connectivity $(Z i)$ and among-module connectivity $(P I)$ [44]. Based on the topological roles in the network, we organized node topology into four categories: network hubs (nodes with high connectivity in the entire network, $Z i>2.5$ and $P i>0.62$ ), module hubs (nodes with high connectivity inside modules, $Z i>2.5$ ), connectors (nodes that connect modules, $P i>0.62$ ) and peripherals (nodes with few outside connections, $Z i<2.5$ and $P i<0.62)[40,45]$.

\section{Shotgun metagenome sequencing and data analysis}

Metagenomic sequencing library preparation was performed using 1 microgram DNA with Illumina (NEB, USA) NEBNext ${ }^{\circledR}$ UltraTM DNA Library Prep Kit as recommended by the manufacturer. The index codes were added to attribute sequence of each compartment sample, and these samples then were purified (AMPure XP system). Agilent 2100 bioanalyzer (Agilent Technologies, CA) was used to check the libraries. Paired-end reads with poor quality (quality score $\leq 38$; base $N>10 \mathrm{bp}$, and the overlap length between adapter and reads was greater than $15 \mathrm{bp}$ ) are filtered. The filtered clean reads (about 11.8-12.5 Gb per sample) were used for metagenomic analyses. The metagenomic assembly was performed using Megahit [46] in default mode. MetaGeneMark (v 2.10) were employed to predict genes from the assembled contigs, and redundancy was removed using CD-HIT Software [47].

For the assembled metagenomes, MetaGeneMark v. 2.10 was used to predict open reading frames, CDHIT v. 4.5.8 was used to construct Unigenes [47], and SoapAligner v. 2.21 was used for quality control. We used DIAMOND combined with the KEGG database (blastp, evalue $\leq 1 e-5$ ) [48] to perform functional annotation on genes related to quorum sensing and cobamide biosynthesis (Additional file 1: Table S1). KO (KEGG Orthology) was divided into higher KEGG categories and KEGG pathways. The gene reading counts were normalized to transcripts per-million (TPM) counts. Statistical enrichment of a given gene between compartments was determined by pairwise comparisons using two-tailed Fisher's exact test, 
with confidence intervals at $99 \%$ significance and Benjamini-Hochberg correction $(P<0.05)$. To get the taxonomical profile of cobamide biosynthesis microbiomes, the sequences were extracted by the seqtk program [49], and subsequently annotated with the Kraken2 program [50].

\section{Results}

\section{Distinctive molecular ecological network structure and connectivity across four mangrove root-associated compartments}

To explore potential microbe-microbe interactions in mangrove roots, we analyzed intra-domain and interdomain microbial interactions based on sequencing datasets of bacterial and fungal communities (Fig. 1a; Additional file 2: Table S2), which were represented as single bacterial and fungal network, and bacterial-fungal interaction (BFI) networks. Across four mangrove root-associated compartments, we obtained a total of 12 microbial co-occurrence networks and their $R^{2}$ of power-law ranged from 0.860 to 0.929 (Additional file 3: Table S3), suggesting that those network connectivity distributions were fitted well with the scale-free property [40].

Multiple network topological metrics consistently revealed that microbial co-occurrence patterns differed profoundly across four root-associated compartments (Fig. 1). The assemblages of three peripheral compartments (non-rhizosphere, rhizosphere and episphere) formed larger and more complex networks with more nodes and edges than that of the endosphere, especially for BFI (Fig. 1b, C). More interestingly, network complexity also differed between intra-domain and inter-domain interaction networks. The BFI networks exhibited a lower average connectivity than their corresponding bacterial and fungal networks in three peripheral compartments (Fig. 1d). However, in the endosphere, a significantly $(P<0.05$, twotailed Fisher's exact test) higher average connectivity was detected in the BFI network (2.986) than in the fungal (2.419) network (Fig. 1d; Additional file 3: Table S3), and more nodes and edges were also observed in the BFI network (426 and 636) than in the single bacterial or fungal networks (210 and 276 for bacteria; 186 and 225 for fungi) (Fig. 1b, c). The results revealed that the endosphere was the only compartment in which the BFI network harbored more intensive interactions than the single bacterial or fungal networks, creating a distinctive niche inside root with the enhanced inter-domain interactions.

We next identified inter-domain microbial assemblages that potentially interacted or shared niches across four root-associated compartments. Four representative BFI networks contained modules with modularity $>0.5$ (Additional file 3: Table S3), and we concentrated on modules with more than 15 nodes. Similar to the overall BFI network structure (Fig. 1a), the number of modules became smaller in transition from the non-rhizosphere, rhizosphere to episphere, but became larger again in the endosphere (Additional file 4: Fig. S1). Notably, we found that module composition did not show clear differences across four rootassociated compartments, in which the proportions of bacteria and fungi were nearly identical (Additional file 4: Fig. S1). The results indicated that the bacteria and fungi contributed similar proportion to the interdomain microbial interactions in the soil-mangrove root continuum. 


\section{Putative keystone taxa across four mangrove root-associated compartments}

On the basis of within-module connectivity $(Z i)$ and among-module connectivity $(P I)$ values, we classified all network nodes into four parts: module hubs, network hubs, peripherals and connectors [11]. Due to the roles in network topology, the taxa detected as module hubs, network hubs and connectors were proposed to be keystone taxa in the complex microbial communities. The results showed that most nodes from each network were peripherals, and no network hubs were identified (Fig. 2). Intriguingly, in three peripheral compartments, more module hubs and connectors were detected in the single bacterial and fungal networks than in the BFI networks (Additional file 5: Fig. S2); however, in the endosphere compartment, the corresponding BFI network harbored more keystone taxa (Additional file 5: Fig. S2), which is consistent with its more complicated networks (Fig. 1a).

To investigate the role of keystone taxa in microbial inter-domain interactions, we focused on BFI networks and their associated keystone taxa across four root-associated compartments. We found that 15,23 , and 13 keystone taxa were observed in the BFI networks of the non-rhizosphere, rhizosphere and endosphere, respectively, but no keystone taxa were detected in the episphere (Fig. 2). Consistently, most of these putative keystone taxa had low relative abundances (Additional file 2: Table. S2; Additional file 5: Fig. S2), suggesting that low-abundant taxa may significantly contribute to mangrove root functions. Further analysis of keystone taxa indicated that fungi accounted for a larger proportion $(60.5 \%)$ in module hubs and connectors of BFI networks than bacteria (39.5\%). Nonetheless, they displayed distinct importance across those four compartments. In the non-rhizosphere and rhizosphere, keystone taxa with top three highest degrees (the number of links for a particular node) were all monopolized by fungi (Additional file 6: Fig. S3). However, in the endosphere, keystone taxa with top three highest degrees belonged to bacteria, and they were affiliated with Vibrio (OTUB_1491), Anaerolineae(OTUB_472) and Desulfarculaceae(OTUB_260) (Fig 2; Additional file 2: Table S2).

\section{Possible microbial interaction mechanisms across four mangrove root-associated compartments}

In order to understand possible mechanisms of microbial interactions across compartments, we investigated the functional profile of root-associated microbial communities by shotgun metagenome sequencing. We focused on functional genes and pathways involved in quorum sensing and cobamide biosynthesis as they have been considered as typical strategies of microbial interactions possessed by various communities $[22,51]$.

Quorum sensing served as a cell-cell communication device, which was exploited by many microflora [51]. Here, we detected quorum sensing circuits with two modules represented by Gram-negative bacteria, and found that genes involved in quorum sensing were unevenly distributed across four continuous compartments (Fig. 3). In module 1, the genes individually related to the production of autoinducer (S)-3hydroxytridecan-4-one (CAl-1) and the mediation of group behaviors, cqsA and IuxR, were highly abundant in the episphere and endosphere, respectively. As the most abundant gene in module $1, t d h$ relates to the production of autoinducer 3,5-dimethyl-pyrazin-2-ol (DPO) and its abundance continuously decreased from the non-rhizosphere to the endosphere. In module 2, las/ and $p q s H$, related to production 
of autoinducer N-(3-oxododecanoyl)-L-homoserine lactone (30C12-HSL) and 2-heptyl-3-hydroxy-4quinolone (PQS), were detected across four compartments, but showed a relatively higher abundance in the endosphere. More strikingly, the endosphere was the only compartment that simultaneously contained $r h / l$ and las $R$, which were involved into the syntheses of autoinducers N-butyryl-L-homoserine lactone (C4-HSL) and the mediation of group behaviors, respectively. The divergent pattern of genes involved in quorum sensing indicated that three peripheral compartments held a greater potential in conducting module 1, whereas module 2 was more likely to be conducted in the endosphere (Fig. 3). Combined with the intensive microbial interactions in the endosphere (Fig. 1a), we infer that module 2 of quorum sensing was closely associated with the enhanceding inter-domain microbial interactions in the endosphere of mangrove roots.

Cobamide, as one of the sharing valuable nutrients, was widely used by microbes to interact with each other [22]. Our metagenome sequencing analysis revealed that among 14 cobamide biosynthesis genes, six genes ( $c y s G, c o b F, c b i B, c o b C, c o b A$, and $c o b D)$ were significantly $(P<0.05$, two-tailed Fisher's exact test) higher in the endosphere than in other three compartments, and they were involved in each of key steps for cobamide biosynthesis, including the tetrapyrrole precursor biosynthesis, corrin ring adenosylation, nucleotide loop assembly and aminopropanol phosphate production (Fig. 4a). Intriguingly, a compartment specificity was detected for anaerobic or aerobic corrin ring adenosylation. Compared to three peripheral compartments, the endosphere harbored a higher abundance of genes associated with aerobic adenosylation ( $c o b G, c o b F$ and $c o b A$ ) and a lower abundance of genes associated with anaerobic adenosylation ( $c b i T$ and $c b i E$ ) (Fig. 4a). Furthermore, the taxonomic composition of all cobamide-related genes across four compartments (Fig. $4 \mathrm{~b}$ ) revealed a high detection frequency of Rhodobacteraceae and Pseudomonadaceae, highlighting their key roles in mediating microbial interactions by producing cobamides. It is important to note that these two families were characterized by a nearly equal detection frequency across four root-associated compartments (Fig. 4b), with a low abundance (2.98\%-6.81\%) in the whole bacterial community (Additional file 7: Fig. S4). Therefore, these results indicated that the intensified microbial interactions in the endosphere were linked to aerobic cobamide biosynthesis performed by specialists (Rhodobacteraceae and Pseudomonadaceae).

\section{Discussion}

Understanding the network structure of microbial communities is essential to decipher their interactions and ecological roles [39]. Through network topology and analysis of genes related to quorum sensing and cobamide biosynthesis, we found niche differentiation of keystone taxa and interaction mechanisms along a soil-mangrove root continuum. Compared to three peripheral compartments, the endosphere exhibited an intensified interaction between bacterial and fungal communities, which was largely attributable to the specific keystone taxa and distinctive interaction strategies.

Given the essential topological role of keystone taxa in microbial networks [52, 53], previous studies have determined the importance of keystone taxa in regulating microbial interactions. In this study, keystone taxa showed a clear divergence between three peripheral compartments and the endosphere. In the 
endosphere, the bacterial keystone taxa with top three highest degrees were likely to have a role in enhancing inter-domain microbial interactions. OTUB_260 was affiliated with the Vibrio genus, and its members were capable of inhibiting fungal phytopathogens by releasing antimicrobial compounds, thereby conducting negative bacterial-fungal interactions [54]. OTUB_472, was affiliated to Anaerolineae, and the members of this family were known to accelerate organic matter degradation when they were stimulated by hyphae exudates of arbuscular mycorrhizal fungi $[55,56]$. Additionally, OTUB_1491 was a member in Desulfarculaceae class, and these typical sulfate-reducing bacteria were reported to act as nutrient providers for fungi and trigger fungal colonization $[57,58]$. It is worth mentioning that the endophytes were capable of forming mixed-community biofilms inside roots [59], which provide a niche for intensive inter- and intra-domain interactions [60]. Taking one keystone taxa (OTUB_260) identified in the endosphere as an example, Vibrio sp. was reported to promote microbial biofilm formation when it communicated with fungi and utilized volatile organic compounds produced by fungi [61]. These results indicated that the intensified inter-domain microbial interactions in the endosphere were related to the specific keystone composition and biofilm formation.

Quorum sensing controls biofilm formation and has been established as a widespread strategy of cellcell communications in microbial communities [62,63]. In the module 1 we detected in this study (Fig. 3), the simultaneous occurrence of $t d h$ and $c q s A$ may be attributable to the weak inter-domain microbial interactions in the episphere. This is because the autoinducers encoded by these two genes were considered to inhibit biofilm formation $[64,65]$ and hence have the potential to hamper microbial cell-cell communications. On the contrary, the module 2 of quorum sensing tended to play a major role in the endopshere, where its involved genes (rhll and lasl) were highly abundant. The autoinducers C4-HSL and 30C12-HSL that were regulated by $r h / /$ and lasl, respectively, were reported to facilitate the establishment of biofilms in the endosphere [66]. These results revealed that the compartment-specific modules of quorum sensing could regulate the levels of biofilm formation, subsequently leading to the divergent microbial interactions between inside and outside mangrove roots. Intriguingly, it has been noted that some of rhizosphere-associated fungi were capable of degrading C4-HSL and 30C12-HSL autoinducers that belonged to the module 2 of quorum sensing [67]. This notion concurs with our observation of less abundant genes related to the module 2 of quorum sensing in the non-rhizosphere, rhizosphere and episphere compartments. Since no fungal taxa from the peripheral compartments could penetrate through the root surface [24], the low abundance of autoinducer-degradative fungi inside roots might explain our finding that the module 2 of quorum sensing dominated in the endosphere and regulated microbial interactions.

Similar to quorum sensing, cobamide has been also widely used as model nutrients for studying microbial interactions [22]. It was established that cobamide mediated microbial interactions through cobamide-dependent metabolism in a metabolic network elicited by cobamide-dependent reactions [22]. As the most well-known cobamide, vitamin B12 produced by bacteria was reported to have stimulatory effect on mycorrhizal growth in plant roots [68], highlighting mutualistic interactions between bacteria and their eukaryotic partners [59]. In this study, we proposed Rhodobacteraceae and Pseudomonadaceae as the key taxa for cobamide synthesis in mangrove roots. Their crucial roles in synthesizing cobamide 
for mediating microbial interactions have been documented in artificial seawater [69]. Through metagenome sequencing analysis across four root-associated compartments, we surprisingly found that the endosphere contained a high abundance of cobamide biosynthesis related genes, and there are two reasons for such enrichment inside plant roots. First, abiotic factors (e.g. oxygen) might contribute to the discrepancy of cobamide biosynthesis between outside and inside roots. Due to the tidal process in mangrove ecosystems [70], a relatively anoxic circumstance probably existed in peripheral compartments [70]. This could explain why we detected a high abundance of $c b i T$ and $c b i E$ encoding for anaerobic corrin ring adenosylation in three peripheral compartments. On the contrary, mangroves were thought to contain large lacunae in the cortex to efficiently transfer internal oxygen, thereby preventing oxygen loss and maintaining aerobic inside the root environment [71]. As a function of such aerobic condition, we observed that the endosphere harbored a high abundance of $c o b G, c o b F$ and $\operatorname{cob} A$ encoding for aerobic corrin ring adenosylation. Second, cobamide utilization can be mediated by quorum sensing molecules. Previous studies have indicated that certain quorum sensing autoinducers, such as $30 \mathrm{C} 12$-HSL from the module 2, would stimulate microbial communities to accumulate cobamides and subsequently promote microbial growth $[72,73]$. In support of this hypothesis, we detected the concurrently enriched genes encoding both the module 2 of quorum sensing and cobamide biosynthesis in the endosphere, which coincided well with intensified interaction between bacterial and fungal communities. Taken together, our study documents the crucial roles of quorum sensing (module 2) and cobamide (aerobic biosynthesis) in mediating the bacterial-fungal interactions in the endosphere of mangrove roots, while future studies may focus on bacterial-fungal interactions at molecular and physiological levels.

\section{Conclusions}

Through topological and metagenomic analyses of four mangrove root-associated microbial communities, we found that bacterial-fungal interactions and their possible mechanisms varied across four mangrove root-associated compartments. Particularly, we proposed a conceptual model of microbial interactions in the mangrove root interior (Fig. 5), where the bacterial keystone taxa, the mediation of quorum sensing (module 2), as well as the aerobic cobamide biosynthesis by Rhodobacteraceae and Pseudomonadaceae might jointly contribute to the intensified bacterial-fungal interactions in the endosphere. Moreover, quorum sensing could indirectly affect microbial interactions by stimulating biofilm formation and mediating cobamide utilization. On the whole, our study provides topological and metagenomic evidence for intensified bacterial-fungal interactions in mangrove root interior.

\section{Abbreviations}

HSL: Homoserine lactone; Non: Non-rhizosphere; Rhi: Rhizosphere; Epi: Episphere; Endo: Endosphere; OTU: Operational taxonomic unit; QIIME: Quantitative insights into microbial ecology; OTUB: Bacterial OTU; OTUF: Fungal OTU; RMT: Random matrix theory; St: Similarity threshold; SRA: Sequence read archive; MENA: Molecular ecological network analyses; KO: KEGG orthology; TPM: Transcripts per-million; BFI: Bacterial-fungal interaction; CAI-1: (S)-3-hydroxytridecan-4-one; DPO: 3,5-dimethyl-pyrazin-2-ol; 
30C12-HSL: N-(3-oxododecanoyl)-L-homoserine lactone; PQS: 2-heptyl-3-hydroxy-4-quinolone; C4-HSL: Nbutyryl-L-homoserine lactone; QS: Quorum sensing.

\section{Declarations}

\section{Ethics approval and consent to participate}

Not applicable

\section{Consent for publication}

Not applicable

\section{Availability of data and materials}

The nucleotide sequences were deposited in Sequence Read Archive (SRA) database under accession numbers PRJNA685020 and PRJNA685297. The metagenome sequencing data were deposited in SRA database with accession numbers of PRJNA613873.

\section{Competing interests}

The authors declare that they have no competing interests.

\section{Funding}

This study was supported by the National Natural Science Foundation of China $(91951207,52070196$, $32000070,41771095)$, the Guangdong Basic and Applied Basic Research Foundation (2019A1515011406), the Fundamental Research Funds for the Central Universities (19lgpy165, 19lgzd28, 19lgpy162), and the Hundred Talents Program through Sun Yat-sen University (38000-18841205, 9931818841205, 38000-18821107).

\section{Authors' contributions}

Zhengyuan Zhou: Investigation, Data curation, Formal analysis, Writing-original draft. Ruiwen Hu: Methodology, Writing-review \& editing. Yanmei Ni: Methodology, Writing-review \& editing. Wei Zhuang: Investigation, Writing-review \& editing. Zhiwen Luo: Investigation, Writing-review \& editing. Weiming Huang: Methodology, Writing-review \& editing. Qiuping Zhong: Methodology, Writing-review \& editing. Qingyun Yan: Methodology, Funding acquisition. Zhili He: Methodology, Supervision, Funding acquisition. Cheng Wang: Conceptualization, Supervision, Funding acquisition, Writing-review \& editing.

\section{Acknowledgements}

Not applicable 


\section{References}

1. Gomes NC, Cleary DF, Calado R, Costa R. Mangrove bacterial richness. Commun Integr Biol. 2011;4(4):419-23.

2. Duran $P$, Thiergart T, Garrido-Oter R, Agler $M$, Kemen $E$, Schulze-Lefert $P$, et al. Microbial Interkingdom Interactions in Roots Promote Arabidopsis Survival. Cell. 2018;175(4):973-83 e14.

3. Ma B, Wang H, Dsouza M, Lou J, He Y, Dai Z, et al. Geographic patterns of co-occurrence network topological features for soil microbiota at continental scale in eastern China. ISME J. 2016;10(8):1891-901.

4. Thatoi H, Behera BC, Mishra RR, Dutta SK. Biodiversity and biotechnological potential of microorganisms from mangrove ecosystems: a review. Ann Microbiol. 2013;63(1):1-19.

5. Banerjee S, Walder F, Buchi L, Meyer M, Held AY, Gattinger A, et al. Agricultural intensification reduces microbial network complexity and the abundance of keystone taxa in roots. ISME J. 2019;13(7):1722-36.

6. Saleem M, Law AD, Sahib MR, Pervaiz ZH, Zhang Q. Impact of root system architecture on rhizosphere and root microbiome. Rhizosphere. 2018;6:47-51.

7. Edwards J, Johnson C, Santos-Medellín C, Lurie E, Podishetty NK, Bhatnagar S, et al. Structure, variation, and assembly of the root-associated microbiomes of rice. Proc Natl Acad Sci 2015;112(8):E911-E20.

8. Bais HP, Weir TL, Perry LG, Gilroy S, Vivanco JM. The role of root exudates in rhizosphere interactions with plants and other organisms. Annu Rev Plant Biol. 2006;57:233-66.

9. Sasse J, Martinoia E, Northen T. Feed Your Friends: Do Plant Exudates Shape the Root Microbiome? Trends Plant Sci. 2018;23(1):25-41.

10. Ofek-Lalzar M, Sela N, Goldman-Voronov M, Green SJ, Hadar Y, Minz D. Niche and host-associated functional signatures of the root surface microbiome. Nat Commun. 2014;5:4950.

11. Shi S, Nuccio EE, Shi ZJ, He Z, Zhou J, Firestone MK. The interconnected rhizosphere: High network complexity dominates rhizosphere assemblages. Ecol Lett. 2016;19(8):926-36.

12. Chen L, Xin X, Zhang J, Redmile-Gordon M, Nie G, Wang Q. Soil Characteristics Overwhelm Cultivar Effects on the Structure and Assembly of Root-Associated Microbiomes of Modern Maize. Pedosphere. 2019;29(3):360-73.

13. Zhang Y, Zheng L, Zheng Y, Xue S, Zhang J, Huang P, et al. Insight into the assembly of rootassociated microbiome in the medicinal plant Polygonum cuspidatum. Ind Crop Prod. 2020;145.

14. de Vries FT, Griffiths RI, Bailey M, Craig H, Girlanda M, Gweon HS, et al. Soil bacterial networks are less stable under drought than fungal networks. Nat Commun. 2018;9(1):3033.

15. Morriën E, Hannula SE, Snoek LB, Helmsing NR, Zweers H, De Hollander M, et al. Soil networks become more connected and take up more carbon as nature restoration progresses. Nat Commun. 2017;8(1):1-10. 
16. Agler MT, Ruhe J, Kroll S, Morhenn C, Kim S-T, Weigel D, et al. Microbial hub taxa link host and abiotic factors to plant microbiome variation. PLOS Biol. 2016;14(1):e1002352.

17. Herren $\mathrm{CM}$, McMahon KD. Keystone taxa predict compositional change in microbial communities. Environ Microbiol. 2018;20(6):2207-17.

18. Miller, Melissa, B., Bassler, Bonnie, L. QUORUM SENSING IN BACTERIA. Annu Rev Microbiol. 2001;55(1):165-99.

19. Kareb O, Aider M. Quorum Sensing Circuits in the Communicating Mechanisms of Bacteria and Its Implication in the Biosynthesis of Bacteriocins by Lactic Acid Bacteria: a Review. Probiotics Antimicro. 2020;12(1):5-17.

20. Banerjee S, Schlaeppi K, van der Heijden MG. Keystone taxa as drivers of microbiome structure and functioning. Nat Rev Micobiol. 2018;16(9):567-76.

21. Shelton AN, Seth EC, Mok KC, Han AW, Jackson SN, Haft DR, et al. Uneven distribution of cobamide biosynthesis and dependence in bacteria predicted by comparative genomics. ISME J. 2019;13(3):789-804.

22. Olga S, Shelton A, Taga M. Sharing vitamins: Cobamides unveil microbial interactions. Science. 2020;369(6499).

23. Alongi DM. Carbon cycling and storage in mangrove forests. Annu Rev Mar Sci. 2014;6:195-219.

24. Zhuang W, Yu X, Hu R, Luo Z, Liu X, Zheng X, et al. Diversity, function and assembly of mangrove root-associated microbial communities at a continuous fine-scale. NPJ Biofilms Micorbi. 2020;6(1):52.

25. Reef R, Feller IC, Lovelock CE. Nutrition of mangroves. Tree Physiol. 2010;30(9):1148-60.

26. Xie X, Weng B, Cai B, Dong Y, Yan C. Effects of arbuscular mycorrhizal inoculation and phosphorus supply on the growth and nutrient uptake of Kandelia obovata (Sheue, Liu \& Yong) seedlings in autoclaved soil. Appl Soil Ecol. 2014;75:162-71.

27. Holguin G, Vazquez P, Bashan $Y$. The role of sediment microorganisms in the productivity, conservation, and rehabilitation of mangrove ecosystems: an overview. Biol Fert Soils. 2001;33(4):265-78.

28. Zhou J, Bruns MA, Tiedje JM. DNA recovery from soils of diverse composition. Appl Environ Microb 1996;62(2):316-22.

29. Tu Q, Deng Y, Yan Q, Shen L, Lin L, He Z, et al. Biogeographic patterns of soil diazotrophic communities across six forests in North America. Mol Ecol. 2016;25(12):2937-48.

30. Peng W, Bo C, Hua Z. High throughput sequencing analysis of bacterial communities in soils of a typical Poyang Lake wetland. Acta Ecologica Sinica. 2017;37(5).

31. Blaalid R, Kumar S, Nilsson RH, Abarenkov K, Kauserud H. ITS1 versus ITS2 as DNA metabarcodes for fungi. Mol Ecol Resour. 2013;13(2):218-24.

32. Marc L, Bolger AM, Axel N, Fernie AR, Lunn JE, Mark S, et al. RobiNA: a user-friendly, integrated software solution for RNA-Seq-based transcriptomics. Nucleic Acids Re. 2012;(W1):W1. 
33. Tanja M, Salzberg SL. FLASH: fast length adjustment of short reads to improve genome assemblies. Bioinformatics. 2011;(21):21.

34. Edgar RC. UPARSE: highly accurate OTU sequences from microbial amplicon reads. Nat Methods. 2013;10(10):996.

35. Caporaso JG, Kuczynski J, Stombaugh J, Bittinger K, Bushman FD, Costello EK, et al. QIIME allows analysis of high-throughput community sequencing data. Nat Methods. 2010;7(5):335-6.

36. DeSantis TZ, Hugenholtz P, Larsen N, Rojas M, Brodie EL, Keller K, et al. Greengenes, a chimerachecked 16S rRNA gene database and workbench compatible with ARB. Appl Environ Microb. 2006;72(7):5069-72.

37. Bengtsson-Palme J, Ryberg M, Hartmann M, Branco S, Wang Z, Godhe A, et al. Improved software detection and extraction of ITS1 and ITS2 from ribosomal ITS sequences of fungi and other eukaryotes for analysis of environmental sequencing data. Methods Ecol Evol. 2013;4(10):914-9.

38. Nilsson RH, Tedersoo L, Ryberg M, Kristiansson E, Hartmann M, Unterseher M, et al. A Comprehensive, Automatically Updated Fungal ITS Sequence Dataset for Reference-Based Chimera Control in Environmental Sequencing Efforts. Microbes Environ. 2015;30(2):145-50.

39. Zhou J, Deng Y, Luo F, He Z, Tu Q, Zhi X. Functional molecular ecological networks. MBio. 2010;1(4):e00169-10.

40. Deng Y, Jiang Y-H, Yang Y, He Z, Luo F, Zhou J. Molecular ecological network analyses. BMC Bioinformatics. 2012;13(1):113.

41. Shannon P, Markiel A, Ozier O, Baliga NS, Wang JT, Ramage D, et al. Cytoscape: a software environment for integrated models of biomolecular interaction networks. Genome Res. 2003;13(11):2498-504.

42. Bastian M, Heymann S, Jacomy M: Gephi: an open source software for exploring and manipulating networks. In: Third international AAAl conference on weblogs and social media. 2009.

43. Newman ME. Modularity and community structure in networks. Proc Natl Acad Sci. 2006;103(23):8577-82.

44. Roger G. Functional cartography of complex metabolic networks. Nature. 2005;7028(433).

45. Olesen JM, Bascompte J, Dupont YL, Jordano P. The modularity of pollination networks. Proc Natl Acad Sci. 2007;104(50):19891-6.

46. Li D, Liu CM, Luo R, Kunihiko S, Tak-Wah L. MEGAHIT: an ultra-fast single-node solution for large and complex metagenomics assembly via succinct de Bruijn graph. Bioinformatics. 2015;(10):10.

47. Fu L, Niu B, Zhu Z, Wu S, Li W. CD-HIT: accelerated for clustering the next-generation sequencing data. Bioinformatics. 2012;(23):3150-2.

48. Moriya Y, Itoh M, Okuda S, Yoshizawa AC, Kanehisa M. KAAS: an automatic genome annotation and pathway reconstruction server. Nucleic Acids Res. 2007;35(Web Server):W182-W5.

49. Shen W, Le S, Li Y, Hu F. SeqKit: A cross-platform and ultrafast toolkit for FASTA/Q file manipulation. PLOS One. 2016;11(10):e0163962. 
50. Wood DE, Lu J, Langmead B. Improved metagenomic analysis with Kraken 2. Genome Biol. 2019;20:257.

51. Milton DL, Hardman A, Camara M, Chhabra SR, Bycroft BW, Stewart G, et al. Quorum sensing in Vibrio anguillarum: characterization of the vanl/vanR locus and identification of the autoinducer $\mathrm{N}$ (3-oxodecanoyl)-L-homoserine lactone. J Bacteriol. 1997;179(9):3004-12.

52. Ma B, Zhao K, Lv X, Su W, Dai Z, Gilbert JA, et al. Genetic correlation network prediction of forest soil microbial functional organization. ISME J. 2018;12(10):2492-505.

53. Fan K, Delgado-Baquerizo M, Guo X, Wang D, Zhu YG, Chu H. Biodiversity of key-stone phylotypes determines crop production in a 4-decade fertilization experiment. ISME J. 2020.

54. Rameshkumar N, Nair S. Isolation and molecular characterization of genetically diverse antagonistic, diazotrophic red-pigmented vibrios from different mangrove rhizospheres. FEMS Microbiol Ecol. 2009;67(3):455-67.

55. Toju H, Sato H. Root-Associated Fungi Shared Between Arbuscular Mycorrhizal and Ectomycorrhizal Conifers in a Temperate Forest. Front Microbiol. 2018;9:433.

56. Cao J, Wang C, Dou Z, Liu M, Ji D. Hyphospheric impacts of earthworms and arbuscular mycorrhizal fungus on soil bacterial community to promote oxytetracycline degradation. J Hazard Mater. 2018;341:346-54.

57. Drake H, Ivarsson M, Bengtson S, Heim C, Siljestrom S, Whitehouse MJ, et al. Anaerobic consortia of fungi and sulfate reducing bacteria in deep granite fractures. Nat Commun. 2017;8(1):55.

58. He H, Fu L, Liu Q, Fu L, Bi N, Yang Z, et al. Community Structure, Abundance and Potential Functions of Bacteria and Archaea in the Sansha Yongle Blue Hole, Xisha, South China Sea. Front Microbiol. 2019;10:2404.

59. van Overbeek LS, Saikkonen K. Impact of Bacterial-Fungal Interactions on the Colonization of the Endosphere. Trends in Plant Sci. 2016;21(3):230-42.

60. Cai P, Sun X, Wu Y, Gao C, Mortimer M, Holden PA, et al. Soil biofilms: microbial interactions, challenges, and advanced techniques for ex-situ characterization. Soil Ecology Letters. 2019;1(3):8593.

61. Cosetta CM, Kfoury N, Robbat A, Wolfe BE. Fungal volatiles mediate cheese rind microbiome assembly. Environ Microbiol. 2020;22(11):4745-60.

62. Padder SA, Prasad R, Shah AH. Quorum sensing: A less known mode of communication among fungi. Microbiol Res. 2018;210:51-8.

63. Kim MK, Zhao A, Wang A, Brown ZZ, Muir TW, Stone HA, et al. Surface-attached molecules control Staphylococcus aureus quorum sensing and biofilm development. Nat Microbiol. 2017;2:17080.

64. Zhu J, Mekalanos JJ. Quorum sensing-dependent biofilms enhance colonization in Vibrio cholerae. Dev cell. 2003;5(4):647-56.

65. Papenfort K, Silpe JE, Schramma KR, Cong JP, Seyedsayamdost MR, Bassler BL. A Vibrio cholerae autoinducer-receptor pair that controls biofilm formation. Nat Chem Biol. 2017;13(5):551-7. 
66. Ozer EA, Pezzulo A, Shih DM, Chun C, Furlong C, Lusis AJ, et al. Human and murine paraoxonase 1 are host modulators of Pseudomonas aeruginosa quorum-sensing. FEMS Microbiol Lett. 2005;253(1):29-37.

67. Uroz S, Heinonsalo J. Degradation of $\mathrm{N}$-acyl homoserine lactone quorum sensing signal molecules by forest root-associated fungi. FEMS Microbiol Ecol. 2008;65(2):271-8.

68. Singh CS, Kapoor A, Wange SS. The enhancement of root colonisation of legumes by vesiculararbuscular mycorrhizal (VAM) fungi through the inoculation of the legume seed with commercial yeast (Saccharomyces cerevisiae). Plant Soil. 1991;131(1):129.

69. Wienhausen GM: Linking the exometabolome of selected organisms of the Roseobacter group to marine dissolved organic matter: a microbiological perspective. Carl-von-Ossietzky Universität Oldenburg; 2018.

70. Wang HT, Su JQ, Zheng TL, Yang XR. Impacts of vegetation, tidal process, and depth on the activities, abundances, and community compositions of denitrifiers in mangrove sediment. Appl Microbiol Biotechnol. 2014;98(22):9375-87.

71. Pi N, Tam NFY, Wu Y, Wong MH. Root anatomy and spatial pattern of radial oxygen loss of eight true mangrove species. Aquatic Botany. 2009;90(3):222-30.

72. Das S, Das S, Ghangrekar MM. Quorum-sensing mediated signals: A promising multi-functional modulators for separately enhancing algal yield and power generation in microbial fuel cell. Bioresour Technol. 2019;294:122138.

73. Johnson WM, Kido Soule MC, Kujawinski EB. Evidence for quorum sensing and differential metabolite production by a marine bacterium in response to DMSP. ISME J. 2016;10(9):2304-16.

\section{Figures}


(a)
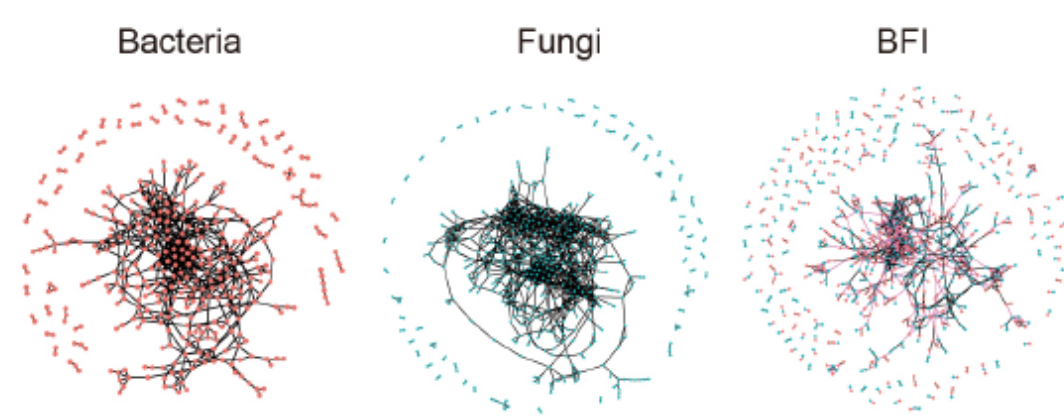

Rhi
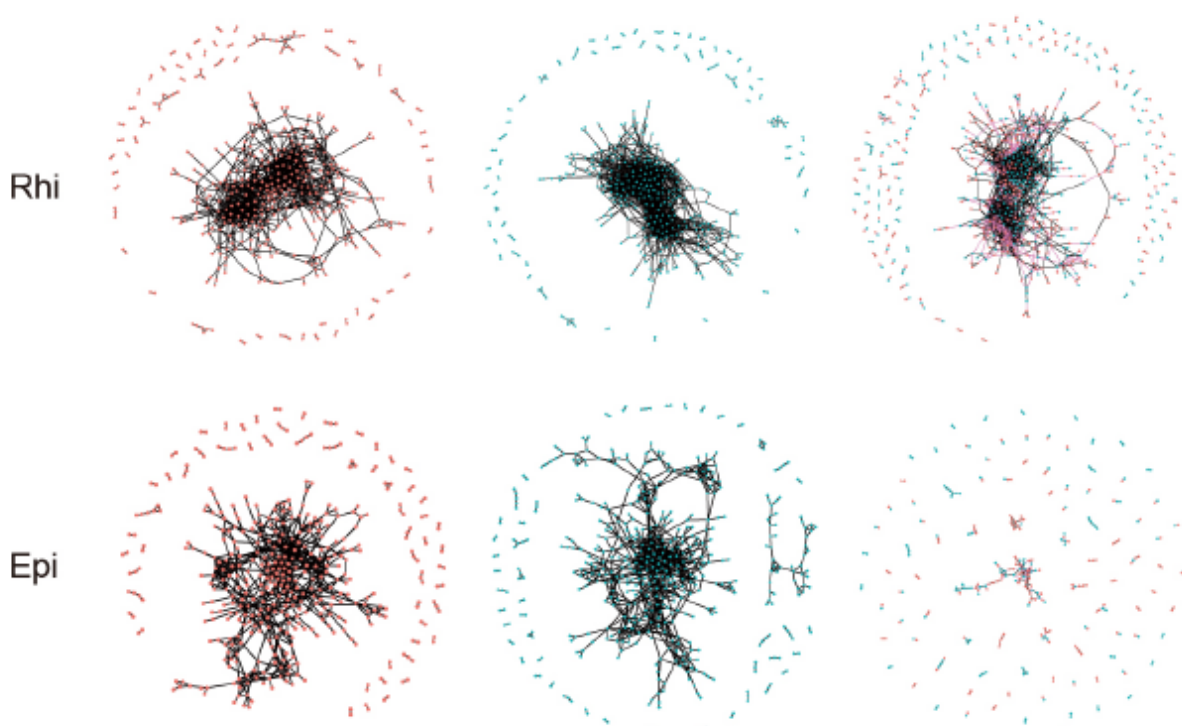

Endo
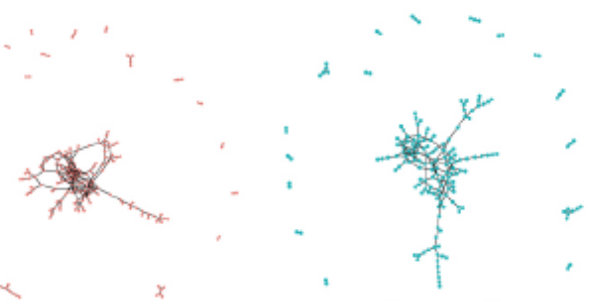

(b)

Number
of nodes of edges Connectivity
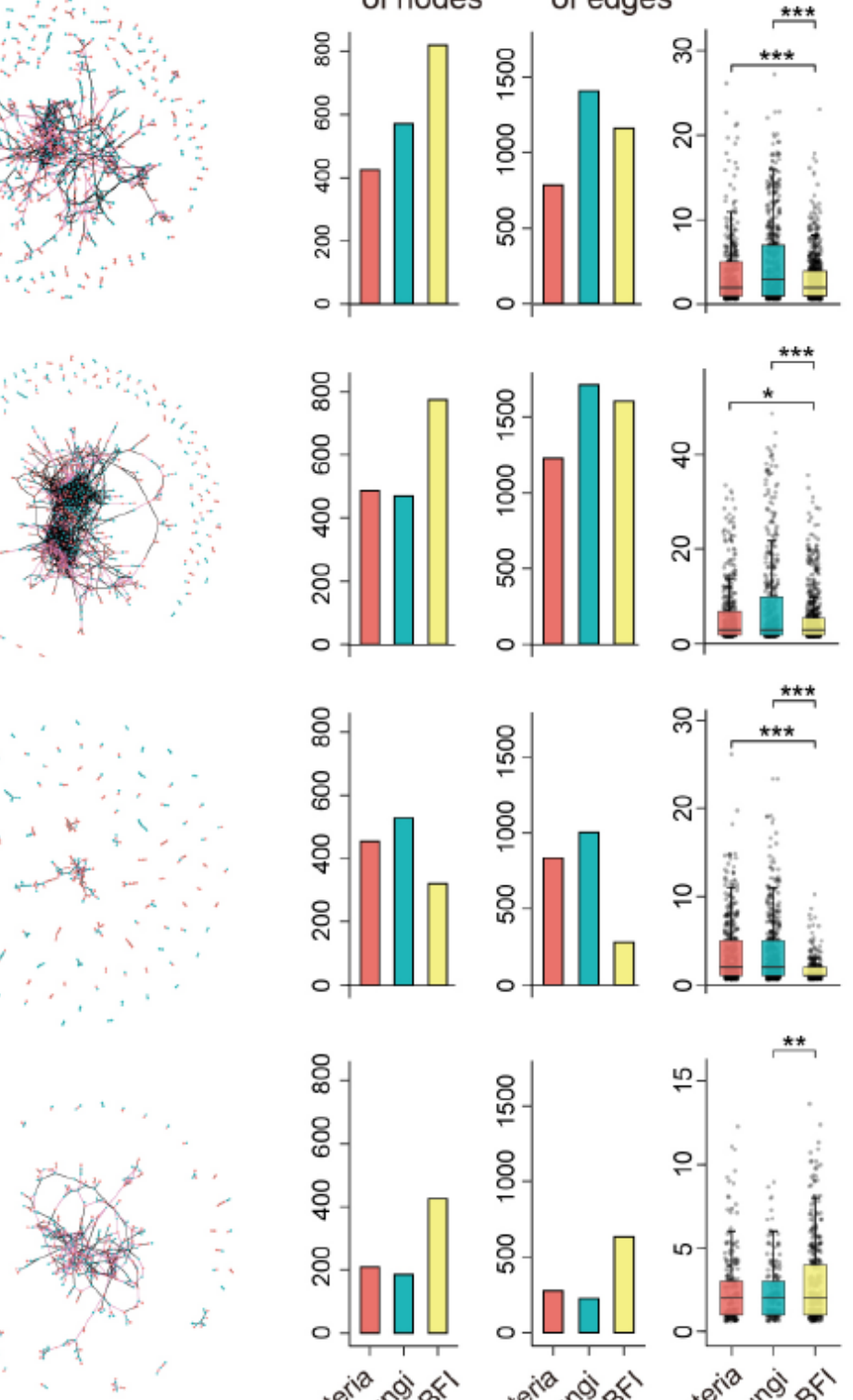

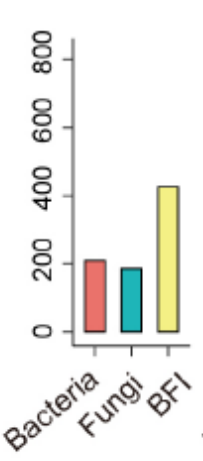

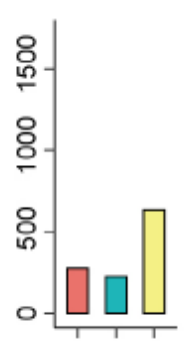

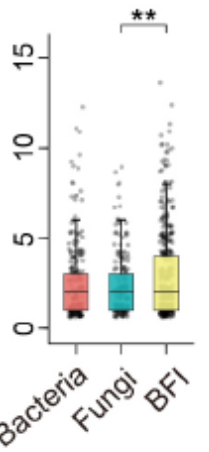

\section{Figure 1}

The profile of intra-domain and inter-domain networks across four root-associated compartments. (a) A representation of bacterial, fungal and bacterial-fungal interaction (BFI) networks across four mangrove root-associated compartments. Each node represents an operational taxonomic unit (OTU), and each edge represents a correlation between two OTUs. Red and blue nodes represent bacteria and fungi, respectively, whereas black and pink edges represent intra-domain and inter-domain interactions, respectively. (b) and (c) show the total number of nodes and edges for each network. (d) The distribution of connectivity harbored by each node in bacterial, fungal and BFI networks. Connectivity is measured by degrees, which refers to the number of edges one node links with. Significance is tested with a two-sided Fisher's exact test: *, $\mathrm{P}<0.05 ; * \star, \mathrm{P}<0.01 ; * \star *, \mathrm{P}<0.001$. 

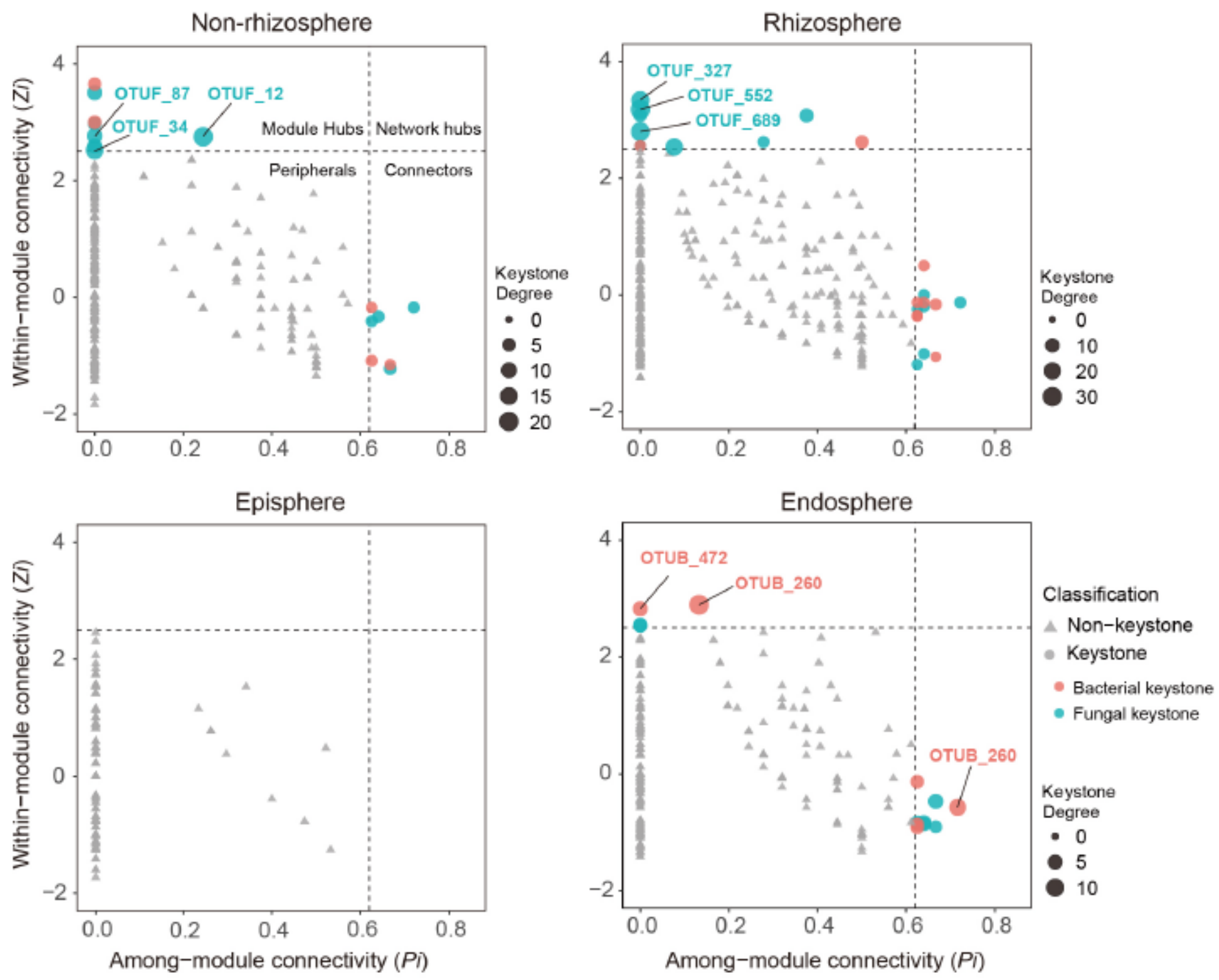

\section{Figure 2}

Topological features of keystone taxa in bacterial-fungal interaction (BFI) networks across four rootassociated compartments. Each bacterial operational taxonomic unit (OTU) is represented as OTUB, and each fungal OTU is represented as OTUF. Modules hubs have within-module connectivity $(\mathrm{Zi})>2.5$, whereas connectors have among-module connectivity $(\mathrm{Pi})>0.62$. Keystone taxa and their domains are classified by shape and color, respectively. The size of each node indicates its degrees; keystone taxa with top three highest degrees in each compartment are labeled. 
Module 1

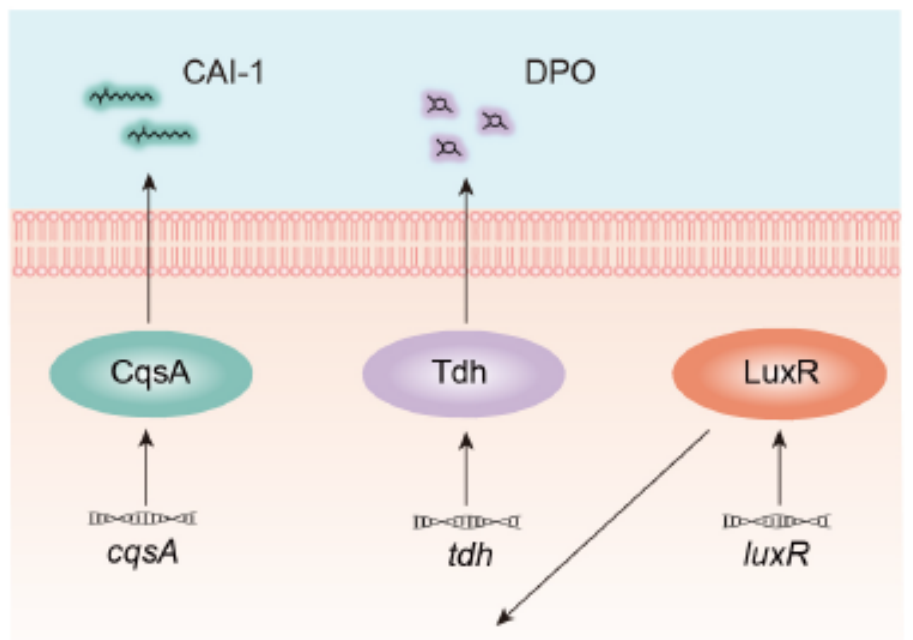

Group behaviors

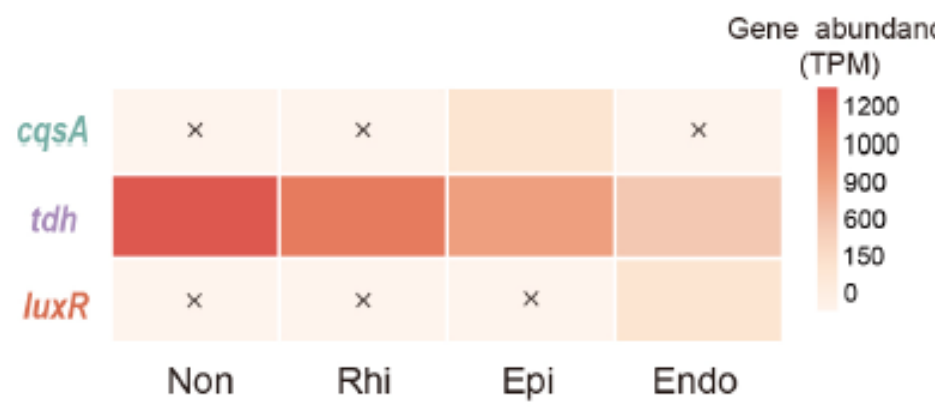

Module 2
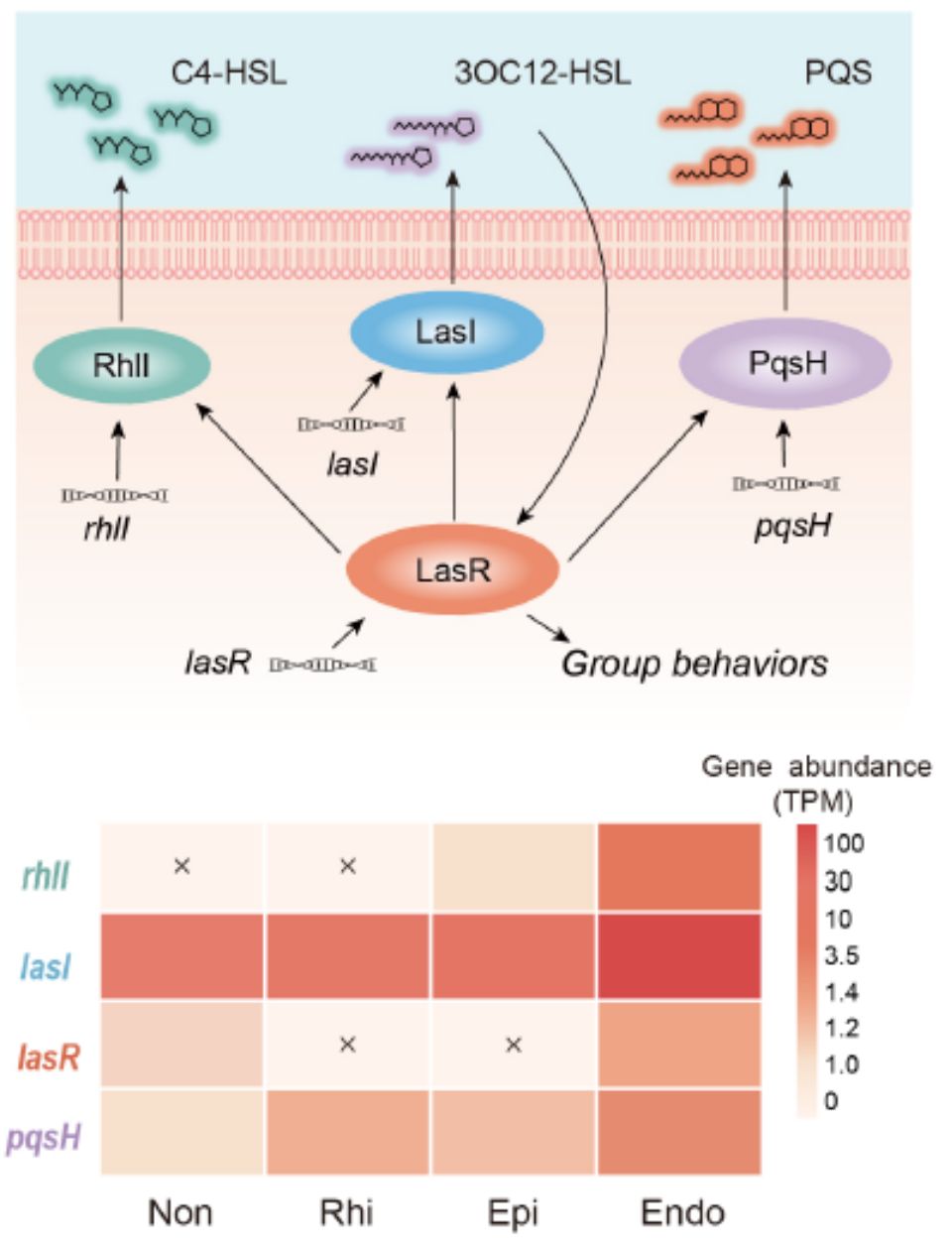

\section{Figure 3}

Profiles of two quorum sensing modules across four root-associated compartments. In module 1, cqsA and tdh encode the autoinducers (S)-3-hydroxytridecan-4-one (CAl-1) and 3,5-dimethyl-pyrazin-2-ol (DPO), respectively, and luxR mediates group behaviors. In module 2, lasl, rhll, and pqsH encode the autoinducers $\mathrm{N}$-(3-oxododecanoyl)-L-homoserine lactone (30C12-HSL), N-butyryl-L-homoserine lactone (C4-HSL), and 2-heptyl-3-hydroxy-4-quinolone (PQS), respectively. lasR encodes the receptor of 30C12-HSL which mediates group behaviors. The relative abundance (TPM, transcripts per-million) of genes belonging to each module across four root-associated compartments is represented by heatmaps, and each undetected gene is marked with a cross. 
(a)

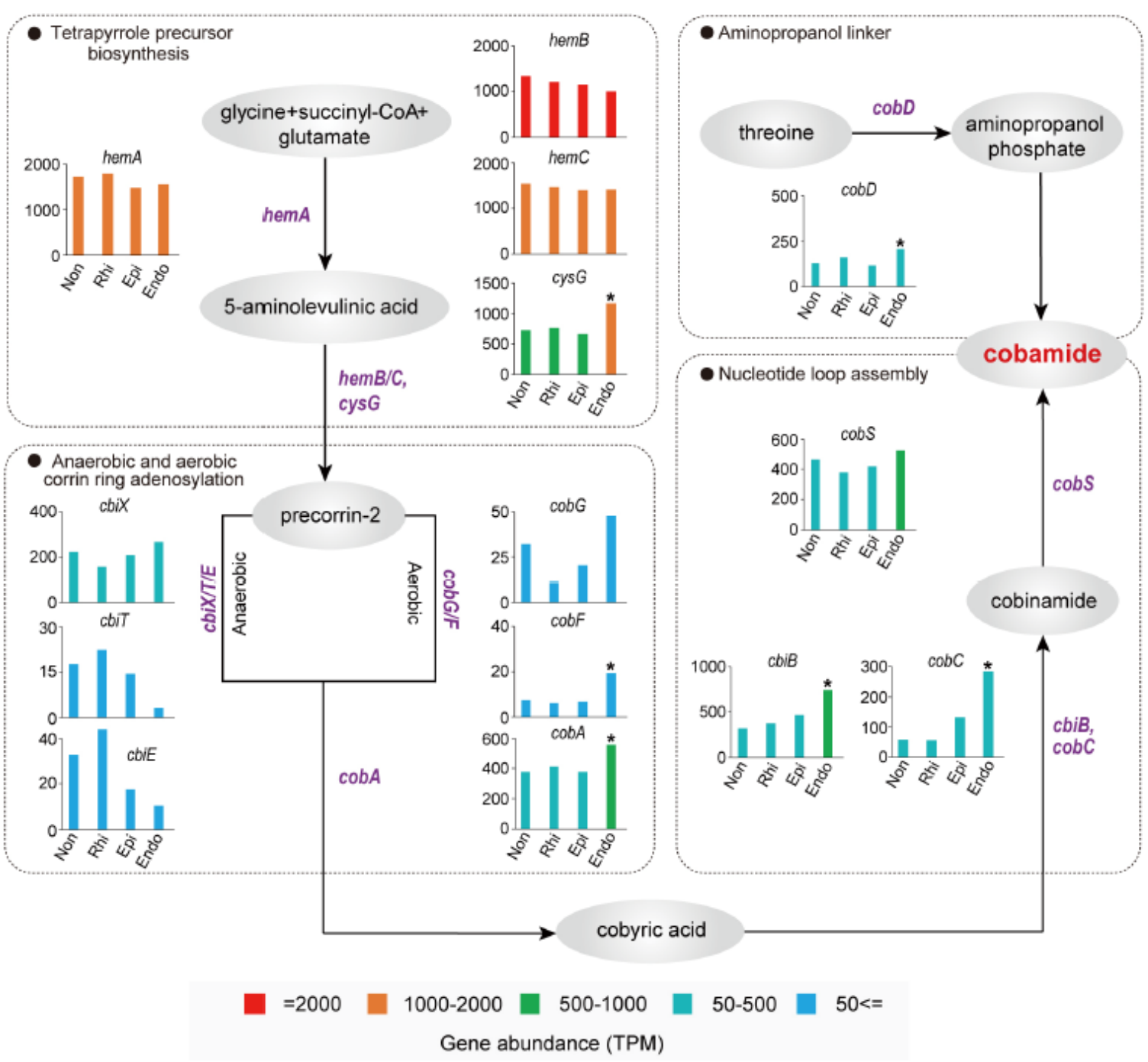

(b)

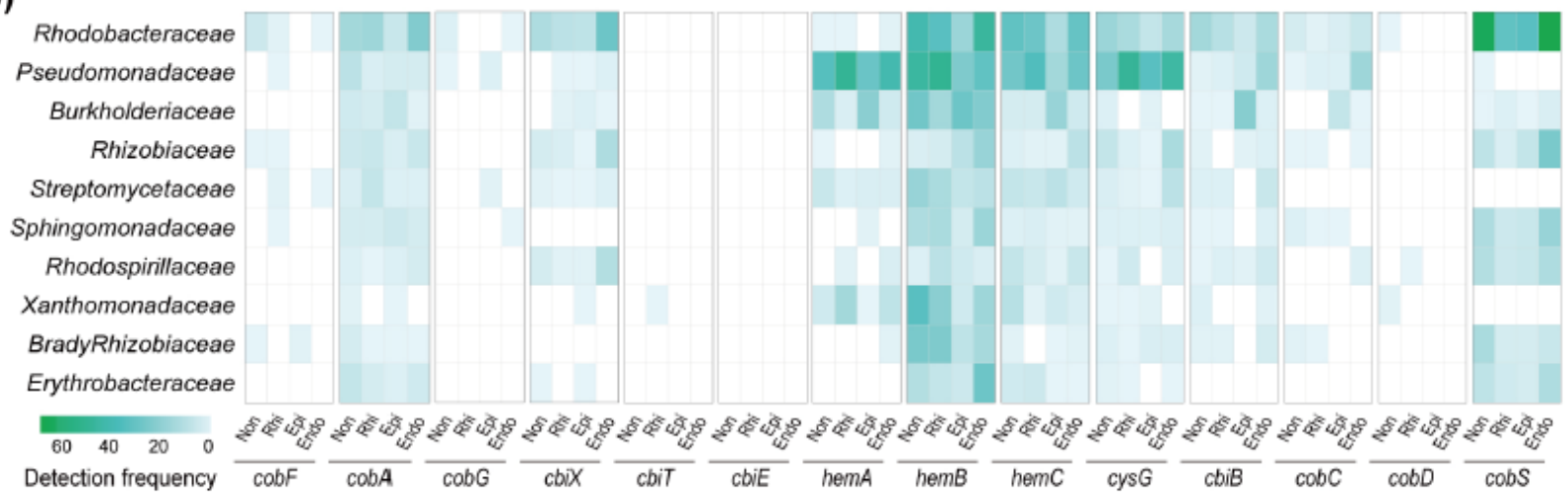

Figure 4

Cobamide biosynthesis pathways and taxonomy profiles of cobamide biosynthesis microbiomes across four root-associated compartments. (a) Each substance is indicated by a gray oval, whereas each functional gene is labeled in purple. The significantly higher TPM (transcripts per-million) value of gene in the endosphere is indicated by an asterisk $(P<0.05$; two-tailed fisher exact test), compared to the three 
peripheral compartments. (b) Bacterial families with the top ten highest detection frequency in cobamide biosynthesis genes are exhibited by a heatmap.

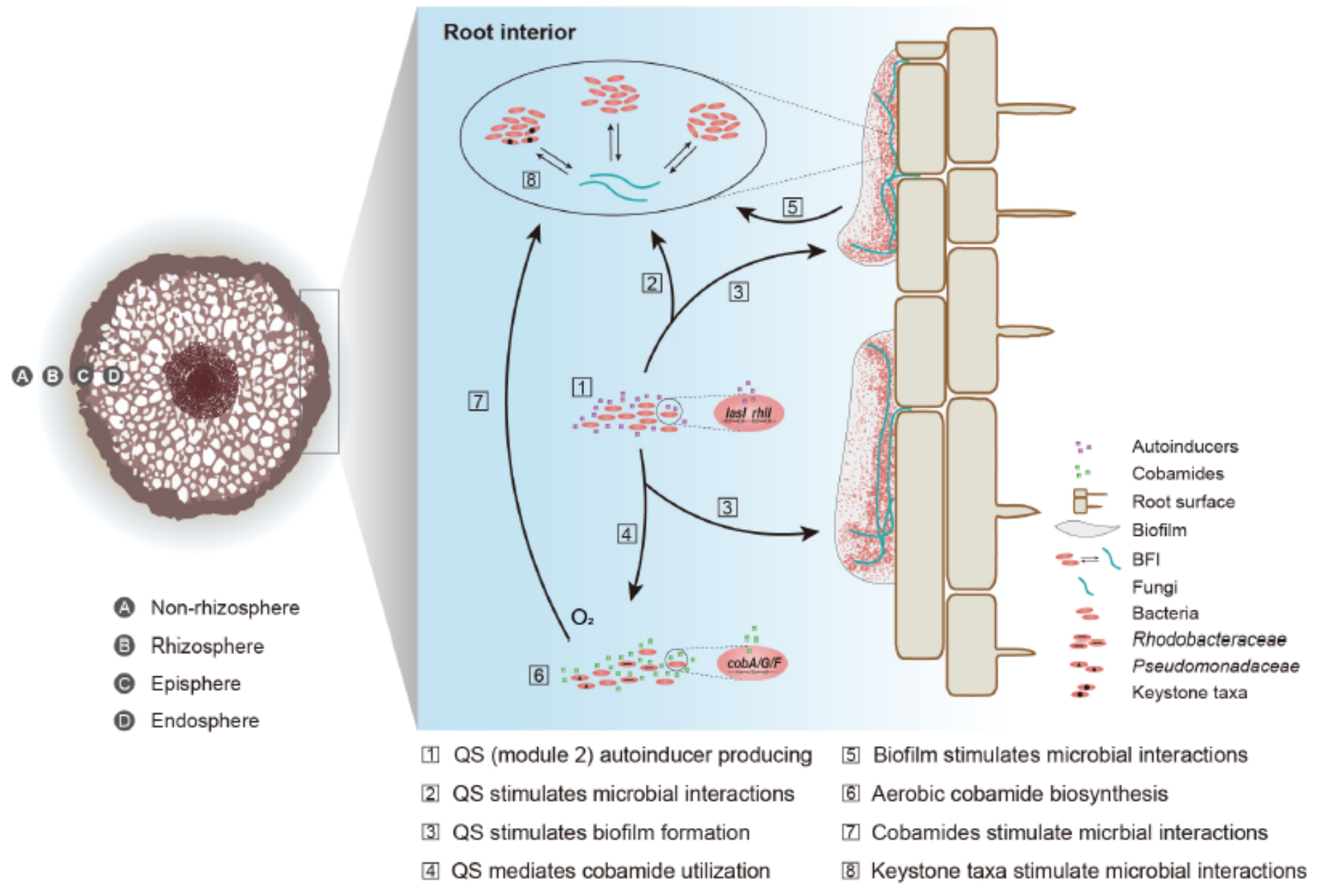

\section{Figure 5}

A conceptual model of the intensified bacterial-fungal interaction (BFI) in root interior.

\section{Supplementary Files}

This is a list of supplementary files associated with this preprint. Click to download.

- Additionalfile1.pdf

- Additionalfile2.pdf

- Additionalfile3.pdf

- Additionalfile4.pdf

- Additionalfile5.pdf

- Additionalfile6.pdf

- Additionalfile7.pdf 